dans les mines métallifères, notamment, l'on est parfois très gênć par l'encombrement des déblais, déchets, ou stériles ; les terrains sont très en pente aux abords des galcries : pour établir des plateformes suffisamment spacicuses, il faudrait Jàtir des murs de soutènemont d'un cubage énorme et cet ubstacle conduit à rouler beaucoup plus loin les déblais sur des terrains plats qui ont plus de valeur ; or, à l'aide des dé blais eux-mêmes on peut fabriquer sur place des gabions Palvis el construire sur le carreau de la mine des plateformes très grandes, soutenues par des murs économiquement édifiés d'après ce système.

Mais ne pouvant nous étendre davantage sur ce sujel, nous ronvoyons les lecteurs qu'il inléresse à la "Société FrancoItalienne de Défenses Fluviales " qui s'est récemment constituée à Grenoble pour l'application en France du Système Palvis, couvert par des brevets.

E.-F. Côte.

\section{NOTE SUR LA RÉGULATION AUTOMATIQUE DE LA VITESSE, DANS L'ACCOUPLEMENT D'UN MOTEUR HYDRAULIQUE ET D'UN MOTEUR THERMIQUE.}

Il nous paraît intéressant, dans certains cas, de régulariser automaliquement la vitesse d'un tel ensemble. Beaucoup de petites usines, qui utilisent une modeste " houille verte", réalisent cet accouplement el voient incessamment changer le mode de fonctionnement de leurs moteurs, soit par la rariation du régime des eaux, soit par la variation du couple résistant de l'usine.

Darss une telle installation, deux éléments sont en présence, qu'il convient de maintenir aussi peu variables que possible :

\section{$1^{\circ}$ La vilesse. $-2^{\circ}$ Le niveau amont de la chute.}

Le premier élément est celui qui donne licu au problèmo proprement dit ; mais pour peu que l'on ail eu à surveiller une semblable installation, on sait que la régulation de la vitesse considérée scule entraine une variation considérable du niveau amont el, par suite, une mauvaise utilisalion de l'eau ou des plaintes de riverains. Nous estimons donc que la régulation complète du premier élément doit comporter en elle-même la régulation du second.

Les trois régimes de meilleur fonctionnement qui peuvent se trouver en un instant donné pour l'installation considéréc sont les suivants :

$I^{\circ}$ Eaux insuffisantes par manque de débit (étiage) :

Vannes de décharges : fermées.

Moteur thermique : ouvert suivant la vitesse.

Turbine : onverte suivant le niveau d'amont.

$?^{0}$ Eaux suffisantes :

Vannes de décharges : ouverles suivant nivean (ou fermées).

Moteur thermique : fermé.

Turbine : ouverte suivant la vitesse.

"Eanx insuffisantes par manque de chute (crues):

Vanne de décharge : ouverte suivant niveau.

Moteur thermique : ouvert suivant vitesse.

Turbine : ouverte en grand.

Parfois, en quelques heures, le régime de l'usine passe le l'une à l'autre de ces circonstances et, plus rarement, par les trois. Dans la presque tolalité des installations actuelles, l'ensemble n'est régularisé que par. Ie régulateur de la machine thermique; le soin d'ouvrir ou de fermer la vanne de garde suivant le niveau, est confié à un ouvrier déjà occupé à une autre besogne, et qui, toujours, accomplit mal cette tâche supplémentaire.

La présente note a pour objet de démontrer la possibilité d'une combinaison de régulateurs satisfaisant à toutes les exigences qui viennent d'être énoncées : les solutions qui sont indiquées sont purement théoriques ct dcmanderaicnt, sans aucun doute, pour êlre réalisées dans de bonnes conditions pratiques, une étude et une mise au point sérieuse de la part des spécialistes : nous aurons alteint notre but si quelques-uns s'intéressent à cette question.

Si l'on se reporte au lableau précédent, on conslate facilement

I' que l'ouverture du moteur thermique dépend de la vitesse seule (Régulateur $\mathrm{N}^{0} \mathrm{I}$ ).

$2^{\prime}$ que l'ouverture des vannes de garde dépend du niveau amont (Régulateur $\mathrm{N}^{\circ} 2$ ).

$3^{\circ}$ que la turbine est ouverte tanlòt suivanl lc nivean amont (Kégulateur $N^{\circ} 4$ ), tantôl suivant la vilesse (Régul. $N^{0} 3$ ).

Ces trois condilions, qui peuvent être appelées conditions de principe, comportent, en outre, pour le bon fonctinnncnient, un certain nombre d'aulres condilions que l'on pout appeler conditions d'accouplentent et qui sont les suivantes :

$\mathrm{I}^{\circ}$ Le régulateur $\mathrm{N}^{\circ} 3$ ne doit pouvoir fermer la lurbine que lorsque le régulateur $\mathrm{N}^{\circ}$ I a complètement fermé la vanne du moleur thermique. Lorsque le réguliteur $\mathrm{N}^{\circ}$ I est cn fonctionnement, la turbine doit etre: ou bien régularisće suivant le niveau amont (Régul. $N^{\circ} 4$ ), ou, dans le cas contraire (excès d'eau), ouverte en grand.

$2^{\circ}$ Le Régulateur $\mathrm{N}^{0} 4$ ne doit pouvoir fermer la turbine que lorsque le Régulateur $\mathrm{N}^{\circ} 2$ a complètement formé la vanne de garde. Lorsque le régulaleur $\mathrm{N}^{0}$ 2) est en fonctionnement, la turbine ou bien doit étre régularisée suivant la vitesse (Régul. $\mathrm{N}^{\circ} 3$ ), ou bien doil être ouverle en grand.

$3^{\circ}$ Les régulatcurs 3 et 4 doivent, en outre, chlo enclanchés de telle sorte que le régulateur de vilesse $\left(\mathrm{N}^{\circ} 3\right)$ cesse d'ouvrir la vanne lorsque cette ouverlure alleint la valeur que détermine le régulaleur $\mathrm{N}^{\circ}$ \&, el que le régulateur de niveau ( $\mathrm{N}^{\circ}$ 2) cesse d'ouviju la vanne lorsque colle ouverlure atleint la valeur que détermine le régulateur $\mathrm{N}^{\circ} 3$, les deux regulateurs restant libres l'un ou l'autre de fermer la vanne.

$4^{\circ}$ Il faut enfin que la cessation du fonctionnement du régulateur $N^{\circ} 3$ remelte en action le rógulateur $N^{\circ}$ I et que la cessation du fonctionnement du régulateur $N^{\circ}$ / mette a fonctionnement le régulateur $\mathrm{N}^{\circ}$ 2.

Nous étudierons sommairement, avant d'exposer une solulion de ce problème :

$I^{\circ}$ Le fonclionnement des régulateurs de vitesse (rappel a'résullats connus) ;

$2^{\circ}$ Le fonclionnement des régulateurs de niveau.

RAPPEL DE QUELQUES PROPRIÉTÉS CONNUES DES RÉGULATEURS DE VITESSE

On sait qu'il existe deux sortes de régulateurs de vitesse (pour les moteurs thermiques, comme pour les moteurs hydrauliques), qui sont : les régulateurs à action directe, et les régulateurs à action indirecte.

Ces régulateurs comportent tous un tachymètre traduisant par un déplacement linéaire une variation de vitesse 
angulaire. La stabilisation du régulateur exige que la posilion du manchon du tachymètre soit une fonction définie de la vitesse ; le plus souvent, le déplacement du manchon $\epsilon=L$ proportionnel à la variation de la vitesse, tout au moins laısqu'on considère un écart faible autour de la vitesse de régime.

Régulaleurs à action directe. - La position de lá varine d'entrée du fluide moteur est fonction de la vitesse ; soit $\omega$ It vitesse au témps $t, \omega+d \omega$ la vitesse au lemps $t+d t$, $S$ l'ouverture maxima du vannage, $s=S-F(\omega)$ l'ouverture correspondante à la vitesse $\omega$, p le couple résistant, $\mu$ le couple moteur, l'équation de d'Alembert s'êcrit :

$$
\frac{\mathrm{d} w}{\mathrm{~d} t}=\frac{\mu_{2}-\rho}{I}
$$

ou, si l'on admet que $\mu$ sodit schsiblement proportionnel à l'ouverture du vannage :

Par suite :

$$
\mu=a[S-F(\omega)]
$$

$$
\frac{\mathrm{d} \omega}{\mathrm{d} t}=\frac{a}{I}\left[\left(S-\frac{p}{a}\right)-F(\omega)\right]
$$

Fxaminons la fonction $F(\omega)$ : elle est nécessairement finie, dans l'intervalle séparant les vitesses $\omega_{\mathrm{m}}$ à pleine charge, et $\omega_{M}$ à vide ; de plus elle est constamment croissante dans ctt intervalle avec $\omega$, puisqu'elle doit exprimer que $s$ décroît Iorsque la vitesse passe de $\omega_{\mathrm{m}}$ à $\omega_{\mathrm{M}}$.

De cette remarque on peut déduire la forme de la variation de $\omega$ avec $t$ dans le cas du régime troublé.

Supposons en effet que le moteur travaille à pleine charge (couple résistant $=R$, vitesse $=\omega_{\mathrm{m}}$ ) et qu'on lui enlève brusquement une partie de sa charge au temps $t=0$ (couple résistant $=\rho$ ) ; la vitesse du nouveau régime (wp sera donnée par : $\quad \mu-\rho=0$

$$
\frac{\mathrm{d} \omega}{\mathrm{d} t}=0 \quad \text { c'est-à-dire : } \quad F\left(\omega_{\rho}\right)=S-\frac{a}{p}
$$

ou :

l.'équation (2) s'écrit :

$$
\frac{\mathrm{d} t}{\mathrm{~d} \omega}=\frac{\mathrm{i}}{\frac{a}{I}\left[\left(S-\frac{\rho}{a}\right)-F(\omega)\right]}
$$

Je dis que cette fonction reste constamment positive entre $\omega_{\mathrm{rn}}$ et $\omega_{p}$. Eh effet, nous avons : $\omega_{\mathrm{m}}<\omega_{p}$.

D'autre part, la fonction dénominateur de (3) n'd pas de racines entr乇 $\omega_{\text {m }}$ et $\omega \rho$; car il faudrait alors prendre cette racine pour valeur de $\omega_{p}$.

Effectuons la substitution de $\omega_{\mathrm{m}}$ à $\omega$ dans l'équation :

$$
\begin{gathered}
\left(\frac{\mathrm{d} \dot{t}}{\mathrm{~d} \omega}\right)_{\omega=\omega_{\mathrm{m}}}^{t=0}=\frac{1}{\frac{a}{I}\left[\left(S-\frac{\rho}{a}\right)-F\left(\omega_{\mathrm{m}}\right)\right]} \\
\text { Or, } \quad F\left(\omega_{\mathrm{m}}\right)=S-\frac{R}{a}
\end{gathered}
$$

Done,

$$
\left(\frac{\mathrm{d} t}{\mathrm{~d} \omega}\right)_{\omega=\omega_{\mathrm{m}}}^{t=0}=\frac{1}{\frac{a}{I}\left[\frac{R-\hat{p}}{\bar{a}}\right]}=\frac{I}{R-p}>0
$$

La fonction $\frac{d \omega}{\mathrm{d} t}$ sera donc constammerrt positive entre $m_{m}$ et wp par suite $t$ croîtra constamment avec $\omega$ dans cet intervalle.

Pour $\omega>\omega_{p}$ la fonction $\frac{\mathrm{d} \omega}{\mathrm{d} t}$ change de signe et $t$ décroît; lorsque os croît wp correspond donc à un lnaximum de $t$.
Je dis que ce maximum n'a pas de valeur finie. En effet, prenons la dérivée seconde :

$$
\frac{\mathrm{d}^{2} t}{\mathrm{~d} \omega^{2}}=\frac{F^{\prime}(\omega)}{\frac{a}{l}\left[\left(S-\frac{a}{\rho}\right)-F(\omega)\right]^{2}}
$$

Or $F(\omega)$ croît avec $\omega$ entre $\omega_{m}$ et $\omega_{M}$. Donc $F^{\prime \prime}(\omega)$ est cons. tamment positive entre $\omega_{\mathrm{m}}$ el $\omega$, par suite la courbe tourne: constamment sa concavité vers l'axe des $t$ positifs, ce quil ne pourrait se produire s'il y avail un maximum de $l$ finis (sauf sil y avail un point de rebroussement dont la tangente serait la droite $\omega=\omega_{p}$; mais pour que cela puisse produire, il faudrait que la courbe ail 3 points à distanc: finic sur la droite $\omega=\omega_{0}$; or, si complexe que puisse être: la fonclion $F(\omega)$ et son intégration, le résultat sera loujours de la forme :

$$
t=\Phi(\omega)
$$

qui indique qu'à une valeur de $\omega$ ne correspond qu'une seule valeur de $t$ réelle).

Quelle que soit la fonclion $F(\omega)$, la courbe de la vilesse

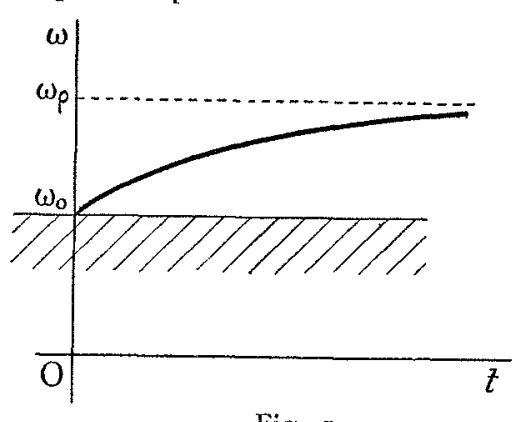

Fig. $\mathrm{r}$ dans les régulateurs directs a toujours la même formc, qui est représentée (lig. (I). Il serait facile de voir que cette forme subsiste encore, lorsque l'on abandonne l'hypothèse de la proportionalite de l'ouverture du vannage: et du couple moteur, pourn. que les variations de l'un el l'autre de ces éléments se

fasscnt dans un sens concordant et suivant une loi continue.

\section{En résumé :}

Dans les régulateurs à action directe (tels que la plupart des régulateurs freins), la vitesse passe insensiblement de la valeur demandée par le premier régime, à la valeur deman: dée par le second régime, sans ondulations ; pendant lo temps du régime troublé, la vitesse reste constamment conprise entre les 2 valeurs extrêmes. On en déduit, en outre, évidemment, que là vitesse d'un moteur ainsi régularisée reste constamment comprise entre sa vitesse à vide ef $\mathrm{sa}$ vitesse à pleine charge.

Régulateurs à action indirecte. - Dans le cas des régulateurs asservis à action indirecte, le second membre de l'équation de d'Alembert peut être, suivant le mode d'action du régulateur sur la vanne, fonction de $t$ seulement ou biell de $\omega$ et de $t$ : le mode de fonctionnement d'un tel régultateur dans le cas où le second membre de l'écquation est fonction de $t$ seulement a été éludié dans La Houille Blanche de février et d'août 1909 , avec quelques hypothèses simplificttrices ; il conduit aux résultats suivants :

La variation de la vitesse entre deux régimes stables, et au cours d'un régime troublé, est une fonction de la variation de charge, de la rapidité d'action du régulateur, do l'inertie du système tournant, de la vitesse du régime, do la puissance de la turbine et du facteur de proportionalit liant le déplacement linéaire du manchon du tachymètrt à la vitesse angulaire Suivant les valeurs de ces divers ét ments, deux cas peuvent se présenter : ou bien la vitess reste constamment comprise, au cours du régime trouble entre les vitesses des deux régimes extrêmes (modérationls ou bien la vitesse ondule en sortant de l'intervalle des tesses extrêmes (ondulations). 
Enfin, lorsque le second membre de l'équation de d'Alembert est fonction de $\omega$ et de $t$, les propriétés des régulateurs doivent être vraisemblablement intermédiaires entre les propriétés des deux cas précédents. Cette catégorie comprend les régulateurs dont l'action est d'autant plus prompte que la variation de vitesse a été plus grande : l'analyse de leur fonctionnement ne semble pas devoir conduire à des résultats simples, par suite des complications d'intégration que présente dans le cas général l'équation de d'Alembert.

\section{RÉGulateurs DU NIVEAU AMONT}

Le régulateur le plus simple du niveau amont est évidemment le déversoir : malheureusement, pour salisfairc à certains règlements, le déversoir doit être considéré comme un apparcil témoin el non comme un appareil régulateur, car le niveau amont ne doit dépasser sensiblemenl la crêle du déversoir que lorsque les vannes de décharge sonl complètenent levóes. Il convienl donc de régler automaliquement la levée de cette vanne.

Considérons le barrage $B_{2}$ ( $\mathrm{ig}$. 2) silué à l'amont du barrage $B_{1}$ sur lequel est placée l'installation à régulariser. Soit $Q$ le débit de la rivière au barrage $B_{2}$ : nous admettons que

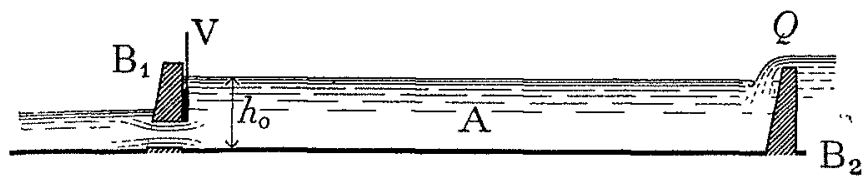

Fig. 2

co débil $Q$ est susceplible de varier dans le temps, mais qu'il ne dépend pas de la hauteur $h_{0}$ de l'eau à l'amonl du barrage $B_{1}$. Soil $S$ la surface de l'eau de la rivière entre les deux barrages. Supposons la vanne ouverte et soil $l$ la largeur de la vamne, $2 \lambda$ la levée.

S'il y a équilibre de régime la quantité d'eau éliminée par la vanne $V$ est équivalente à celle qui passe en $B_{2}$ (en faisant abstraction des infiltrations, des affluenls, ou des canaux de dérivation). Par suile, en régime, nous aurons :

$$
Q=2 m \lambda l \sqrt{2 g\left(h_{0}-\lambda\right)}=K \lambda V \sqrt{h_{0}-\lambda}
$$

Supposons que $Q$ vienne à augmenter, le niveau $N$ s'accroît jusqu'à l'instant où :

$$
Q^{\prime}=2 m \lambda l \sqrt{2 g(h-\lambda)}=K \lambda V \overline{h-\lambda}
$$

ce qui donne pour $h$ :

$$
h-\lambda=\left(h_{0}-\lambda\right) \frac{Q^{\prime 2}}{Q^{2}}
$$

ou cncore, en prenant pour origine des niveaux l'axe de l'orifice de la vanne :

$$
\frac{H}{H_{0}}=\frac{Q^{\prime 2}}{Q^{2}}
$$

Il y a donc là un phénomène de régulation automatique, puisque lo hiveau $h$ se limile de lui-même, mais cette ré-

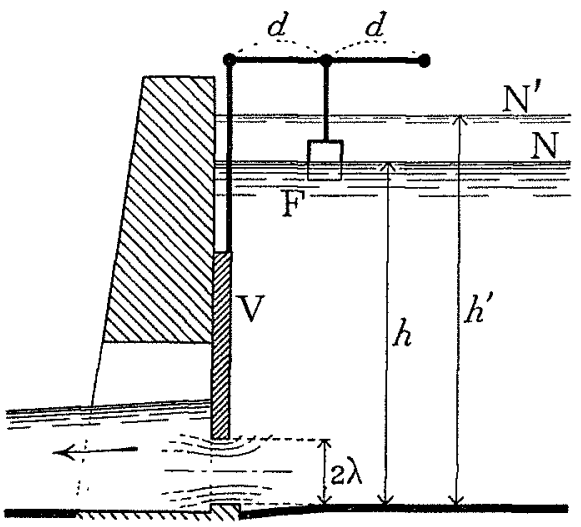

Fig. 3 gulation est absolument illusoire ; il suffit de remarquer en effet que, si le débit de l'eau double, la hauteur $H$ se quadruple, pour comprendre l'insuffisance d'un tel procédé.

Supposons qu'au lieu d'être maintenue constante, l'ouverture de la vanne croisse proportionncllement au niveau, ce qui peut être schómatiquement représcnté en supposant la vanne liée à un flotteur F (fig. 3).

Considérons un instant $t$, soit $h$ le niveau amont, $d h$ la variation de ce niveau pendant le temps $d t$, nous avons :

$$
\lambda=a\left(h-h_{0}\right)
$$

Ja vanne élant fermće lorsque le niveau prend la valeur $h_{0}$. Le volume d'eau passant par l'orifice de la vanne dans l'instant $d t$ sera :

$$
q d t=2 m l a\left(h-h_{0}\right) \sqrt{2 g\left[h-a\left(h-h_{0}\right)\right]} d t
$$

et si nous nous plaçons dans le cas parliculier où la vanne serait liće au flotteur de telle sorte que $a=\mathbf{I}$ (cas de la fig.3) :

$$
\begin{aligned}
q d t & =2 m l a\left(h-h_{0}\right) \sqrt{2 g h_{0}} d t \\
& =K\left(h-h_{0}\right) d t
\end{aligned}
$$

D'autre part, le volume d'eau passant au déversoir $B_{2}$ pendant le temps $a l$ est :

\section{$Q d t$}

la différence représentera l'eau emmagasinée culre les deux barrages, différence qui aura également pour expression :

$$
\begin{gathered}
S d h \\
\text { par suite : } \quad S d h=Q d l-q d l \quad\left(\text { ou }: \frac{d h}{d t}=\frac{Q-q}{S}\right) \\
S d h=Q d t-K\left(h-h_{0}\right) d t
\end{gathered}
$$

ou encore

$$
\frac{d h}{d t}=\left(Q+K h_{0}\right)-K h
$$

ou encore

$$
\frac{d h}{d t}=\frac{Q+K h_{0}}{S}-\frac{K}{S} h .
$$

équalion qu'il suffil de rapprocher de l'équation (a) pour constater leur parfaite identité de forme. Toules les propriétés connues concernanl les régulateurs de vilesse à aclion directe pourront done s'appliquer au régulalcur de niveau ainsi établi.

Si de plus l'on rapproche l'équation (3 bis) de l'équation de d'Alembert :

$$
\frac{d \omega}{d t}=\frac{\mu-p}{I}
$$

on constate que $S$ joue sensiblement le même rôle que $I$ el que par suite la régulation scra d'autant plus facile que la surface du lit de la rivière sera plus grande, ce qu'il était facile de prévoir à priori.

Dans un tel système, si $Q$ est le débit minimum correspondant à une hauteur $h$ el $Q$ ' lo débit maximum correspondant à $h$; nous aurons évidemment

$$
\frac{h^{\prime}-h_{0}}{h-h_{0}}=\frac{Q^{\prime}}{Q}
$$

ou, en prenanl pour origine le niveau $h_{0}$ :

$$
\begin{gathered}
h^{\prime}-h_{0}=H^{\prime} \\
h-h_{0}=H \\
H^{\prime}=\frac{Q^{\prime}}{Q}
\end{gathered}
$$

les hauteurs seront donc proportionnelles aux débits et non plus comme précćdemment aux carrés des débils.

La régulation sera ainsi beaucoup plus efficace comme le démontre tout à fait l'exemple suivant : 
Supposons une yanne ouverte de $0^{\mathrm{m}} 40$ sur une largeur $l$, soit I $\mathrm{m}$. la hauteur du niveau comptée à partir de l'axe de l'ouverture de la vanne, niveau correspondant au débit $Q$; supposons que le débit devienne $2 Q$; si $H$ est la hauteur du niveau correspondant à ce débit

$$
\frac{H}{\mathrm{r}}=\frac{4 Q^{2}}{Q^{2}}=4
$$

Par suile, si l'ouverture reste la même, le niveau sera de 4 $\mathrm{m}$. au lieu de 1 mètre.

Supposons au contraire que la vanne suive le niveau de l'eau, nous aurons, en comptant les niveaux à partir du fond :

$$
\begin{gathered}
h_{0}=\mathrm{o}^{\mathrm{m}} 8 \mathrm{o} \\
h-h_{0}=\mathrm{x}, 20-\mathrm{o}, 80=\mathrm{o}^{\mathrm{m}} 40
\end{gathered}
$$

le nouveau niveau $h$ ' donnera :

ou :

$$
\begin{gathered}
\frac{h^{\prime}-h_{0}}{h-h_{0}}=\frac{2 Q}{Q}=2 \\
h^{\prime}-h_{0}=0,80 \\
h^{\prime}=\mathrm{I}, 60
\end{gathered}
$$

La variation réelle du niveau aura donc été dans le premier cas de $3 \mathrm{~m}$. et dans le second de $\mathrm{o}^{\mathrm{m}} 4 \mathrm{o}$ seulement.

Dans les régulateurs de cette nature, la différence entre les niveaux correspondant au débit maximum et au débit minimum ne peul être supprimée, pour la même raison que dans les régulateurs de vitesse directs il n'est pas possible de supprimer la différence de vitesse à vide et à pleine charge. Dans ces régulateurs, en effet, l'ouverture de la vanne est une fonction de la vitesse : pour que cette ouverture prenne des valeurs diverses, il faut donc que la vitesse prenne des valeurs diverses suivant la charge.

Il ne paraît pas pratique de réaliser pour une vanne de déchąrge un régulateur de ce genre : ces vannes sont le plus couvent lourdes, encombrantes et difficiles à manier, II est plus simple, croyons-nous, d'avoir recours aux régulateurs indirects.

Régulateur indirect du niveau amont. - Notons d'abord qu'un simple flottcur F (fig. 4) traduit immédiatement en déplacements linéaires le phénomènc à régulariser ; il suffit donc d'assimiler ce flotleur au manchon d'un tachymètre et tous les organes d'asservissement, tous les perfectionnements apportés aux servo-moteurs, dans les régulateurs de yitesse, seront applicables à la régulation du niveau amont, la fermeture ou l'ouverture do la vanne étant produite par un levier I. dont le point d'appui $O$ scra asservi à la position de la vanne. (Pour simplificr la gravure, nous avons admis que le levier $\mathrm{L}$ actionnait la vanne par fermeture

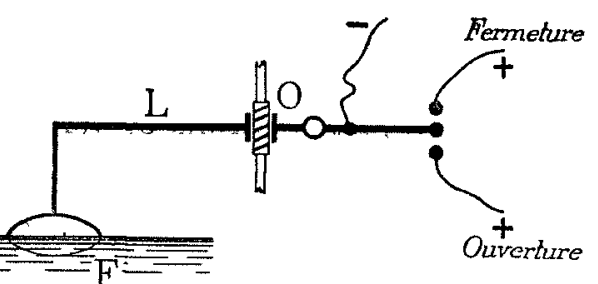

dans le sens convenable d'un circuit électrique). Un tel régulatcur fonctionnera donc de la mème façon qu'un régulateur de vitesse. En particulier, suivant le mode d'action du servomoleur commandant la vanne, deux cas pourront se présenter :

\section{L'équation}

$$
\frac{d h}{d t}=\frac{Q-q}{S}
$$

analogue de l'équation de d'Alembert dans la régulation des vitesses, sera fonction ou bien de $t$ șeulement, ou bien à la fois de $h$ et de $t$.

Dans le premier cas, la variation du niveau suivra une loi analogue à celle qui a été étudiée dans les mêmes circonstances pour la régulation de la vịtesse ; si l'on a sain toutefois de réaliser pratiquement les hypothèses simpliffcatrices dont il a été parlé à propos de cette étude.

En oulre, ce qui a élé dil concernant les deux modes d'ar. tion possible de la correction de réglage, subsịstera dạns la régulation des niveaux amont : modération ou ondula' tion, avec cetle différence toulefois que le débit $Q$ varier toujours lentement, ce qui fait que, dans l'application pra tique, on ne rencontrera vraisemblablement jamais d'ondulation dans la hauteur du niveau, le niveau passant purement et simplement de la valeur correspondante au débit $Q$ à la valeur correspondante au débit $Q^{\prime}$ d'une façon progressive, la hauteur de ces deux niveaux pouvant d'ailleurg différer très peu.

De ce qui précède résulte surtoul, par analogie avec la régulation de la vitesse, que si l'on veut une variation relativement faible du niveau amont entre les régimes de débit minimum et de débil maximum, il conviendra de choisir un régulateur à servo-moteur asservi, régulateur pour le. quel celte variation peut être réduite pour ainsi dire auttant qu'on le désire. Il n'y aura pas lieu de craindre une variation du niveau amont trop grande en régime troublć.

\section{RÉGULATION DU SYSTÈME MOTEUR-THERMIQUE MOTEUR-HYDRAULIQUE}

La figure (5) indique le schéma de la solution proposéé. Le Régulateur $\mathrm{N}^{\circ}$ I est le régulateur indirect de vitesse de la machine thermique, $A_{1}$ est le tachymètre du moteur thermique dont le réglage sera tel que le manchon sera à să buttée supérieure pour une vitesse $\omega_{1}$ et à la buttée inférieure pour la vitesse $\omega_{\mathrm{m}}$, la plus faible que l'on admette en régime.

$O_{1}$ sera le point d'appui asservi au déplacement de ta vanne (ou détente) du moteur thermique. Un déplacement du manchon aura pour effet de fermer le courant de la soulce électrique $D$ sur l'un des enroulements $\Omega_{1}$ ou $F_{1}$ quil eux-mêmes actionneront mécaniquement la vanne dans lé sens demandé par le tachymètre ; le déplacement du levier sera d'ailleurs aussitôt corrigé par le déplacement du point $\mathrm{O}_{1}$ lié à la vanne. Lorsque la vanne sera complètement fermée, un contact s'établira entre $a_{1}$ et $b_{1}$, et fermera la li: gne $\beta_{3} a_{1}$ sur $b_{1} \mathrm{~F}_{3}$.

(3) est le régulateur de yitesse de la turbine. $A_{3}$ le tachy. mètre dont le manchon est à sa buttée inférieure pour la vitesse $\omega_{1}$ et à sa luttée supérieure pour la vitesse $\omega_{M}$ la plus forte qui soit admise en régime : $\mathrm{O}_{3}$ est le point d'apput asservi, $\alpha_{3} \beta_{3}$ les contacts déclanchant la fermeture ou l'our verture de la turbine au moyen des enroulements $F_{3}$ et $\Omega_{g}$ En outre lorsque $A_{3}$ est à sa buttée inférieure (vitesse $\omega_{1}$ ) la ligne $\Omega_{3} \gamma_{3}$ est fermée sur $\delta_{3} \beta_{4}$.

(2) est le régulateur de niveau amont agissant sur la vanne $\mathrm{B}_{2}$ le flotteur, $\mathrm{O}_{2}$ le point d'appui asservi, $\alpha_{2} \beta_{2}$ le contacts fermant ou ouvrant la vanne de décharge par les enroulements $\mathrm{F}_{2} \Omega_{2}$. Le flotteur $\mathrm{B}_{2}$ est à sa buttée supérieftre pour la hauteur $\mathrm{H}_{\mathrm{M}}$, la plus grande admise pour le nivealf amont en régime, et à sa buttée inférieure pour une hallteur plus faible $\mathrm{H}_{\mathrm{b}}$, Lorsque la vanne est fermée complètement, elle établit un contact électrique entre $a_{2}$ et $b_{2}$.

(4) est le régulateur de la turbine, agissant suivant le n: veau amont, $\mathrm{O}_{1}$ le point fixe asservi, $\mathrm{B}_{4}$ le flotteur qui 
à sa buttée inférieure pour la hauteur $H_{m}$ la plus faible admise en régime, et à sa buttée supérieure pour la hauteur $H_{1}$ Lorsque le llotleur est à sa bullée supéricure, il ferme la ligne $\alpha_{3} \gamma_{4}$ sur $\delta_{4} \Omega_{3}$, le levier régulateur ferme ou ouvre la vanne de la turbine dans le sens convenable au moyen des contacls $\alpha_{1}$ et $\beta_{4}$. Nous supposerons en outre que les contacts $\alpha_{1} \beta_{1}, \alpha_{2} \beta_{2}, \alpha_{3} \beta_{3}, \alpha_{4} \beta_{4}$ sonl établis avec le minimum de jeu possible (condition de sensibililé : voir Houblle Blanche de février rgog) ; les deux points $\gamma_{3} \partial_{3}$ seront considérés comme les bultcés extrêmes du manchon $m_{4}$ el $\gamma_{4} \partial_{4}$ les butlées extremes do $B_{4}$. Par contre, les contacts en $\alpha_{1} \beta_{1}, \alpha_{2} \beta_{2}, \alpha_{3} \beta_{3}$, y a d'arrangements de 3 lettres prises 2 à 2 , soit 6 . Nous itudierons le fonctionnement des régulateurs dans chacun de ces 6 cas et, si le fonctionnement en est satisfaisant, le problème sera résolu.

(I-II). - Dans le régime I (eau insuffisante par manque de débit), la vanne de garde est fermée, le niveau compris entre $H_{1}$ et $H_{m}, B_{2}$ à sa buttée inférieure, $B_{4}$ en action, le contacl $a_{2} b_{2}$ établi, les contacls $\alpha_{2} \beta_{2}$ coupés aulomaliquement par l'asservissement de $O_{2}$. Le régulateur (1) est en fonctionnement, le contact $a_{1} b_{1}$ coupé, la vilesse comprise entre $\omega_{i} \mathrm{Cl} \omega_{n 1}$, par suite le manchon de $A_{3}$ à sa bultée infólieure fermant le conlact $\partial_{3} \gamma_{3}$. Dans ces condilions, les circuits I) $\left.\alpha_{4} a_{2} b b_{2} F_{3}\right)$ on $D \beta_{4} \delta_{3} \gamma_{3}\left(Q_{3}\right.$ I) sont ouverts ou formés à la dremande de $B_{4}$, la larbine clant ouverte à la demande du niveau supposolís que leau deviemme suffisanle par diminufion du couple résistanl, la,vilesse s'accélère, $\Lambda_{1}$ ferme la vanue du motem thermique, le conlact $a_{1} b_{1}$ s'élablit ì cel instant, la vitesse contimuant à croîlre passe dans l'intervalle (1) $\omega_{\mathrm{m}}$ el le manchon de $A_{3}$ se live, compant le contact $z_{3} \gamma_{3}$, cl élablissant lo circuit D $\beta_{3} a_{1}$ $b_{1} F_{3} D$ qui ferme la vanne de la turbine. Dans ces condilions, le niveau compris entre $\boldsymbol{I}_{1}$ et $\mathrm{H}_{\mathrm{m}}$ monte, mais $\mathrm{B}_{4}$ demandant vainement l'ouverture (ca le circuit est coupé an $\delta_{3}$ ) finit par se fixer à sa bulléo supérieure et ferme lo contact $\varphi_{1} \partial_{1}$. Le niveau passe donc dans l'inlervalle $H_{i} \quad H_{n 1}$ mais alors $B_{2}$ entre en fonctionnement el ouvre la vanne de garde à la demarde du niveau en coupant le conlact $a_{2} b_{2}$. En dernier lieu on voit que ( 1 ) et (1) n'agissent plus, (3) et (2) agissant seuls ; une série d'enclenchements inverses se produiraient dans lo passage de II à 1 .

II-III. - Le régime étant ćtabli comme il vienl d'être dil, supposons qu'il advienne une crue subile qui nous amèné Les pelits cerrles blanes sur les leviers indquenl l'isolement électrique des diverses branches de ces leviers. $z_{4} \beta_{4}$ seront supposés ne pas former butlér et so faire sans frollment appréciable.

La figure montre enfin comment sont groupés ct reliés électriquement les différènts éléments énumérés ci-dessus.

Dans ces conditions, je dis :

$I^{\circ}$ que la vitesse de régime restera constamment comprise entre les deux valeurs $\omega_{\mathrm{m}}$ et (1)

$2^{\circ}$ que le niveau amont de régime restera constamment compris entre les deux hauteurs $\mathrm{H}_{\mathrm{m}}$ et $\mathrm{H}_{\mathrm{M}}$ (jusqu'à concurrence de la capacilé d'écoulement de la vanne de garde).

Nous avons vu précédemment que 3 cas de groupement des appareils pouvaient normalement se présenter. II $y$ aura donc autant de passage d'un groupement à l'autre qu'il au régime $\mathrm{N}^{\circ}$ III. Le niveau de l'eau s'accroissant sollicite l'ouverture de la vanne de garde. Celle-ci s'ouvre en grand ct débraie automatiquement son servo-moteur à fin de course par l'asservissement de $\mathrm{O}_{2}$. La chule diminuant, la vitesse diminue et $\mathrm{A}_{3}$ ouvre la turbine jusquáa moment où celle-ci étant ouverte en grand débraie automatiquement les derax régulateurs (3) et (1) ; Iorsque la vitesse continue à décroître, elle tombe dans l'intervalle $(1)_{1}(1)$ m el $\Lambda_{1}$ entro en fonctionnement. Une série d'enclenchements inverses se produit dans le passage de III à II.

III-I. - Ce cas ne se rencontrera probablement jamais sans que le cas $N^{\circ}$ II se place comme intermédiaire. Cependant il n'est pas impossible, et il convient évidemment que 
lè régulateur puisse satisfaire aúx contditions de ce passage.

Prenons donc l'ensemble dans l'état où il vient d'être laissé et supposons une diminution subite de l'eau, nous ramenant au cas $\mathrm{N}^{\circ} \mathrm{I}$. Le niveau baissant rapidement, $\mathbf{B}_{2}$ ferme la vanne de garde, rétablit le conlact $d_{2} b_{2}$, ct la vanne à lin de course débraic automatiquement son régulateur ; le nireau passe dáns l'intervalle $\mathrm{H}_{i} \mathrm{H}_{\mathrm{m}} ; \mathrm{B}_{4}$ devient actif et ferme la turbine ; la vitesse restant entre $\omega_{\mathrm{i}}$ et $\omega_{\mathrm{m}}$, aucun changement rie se produit dans le fonctionnement des régulatcurs (r) el (3).

Une série d'enclenchements inverses se produit dans le passage de I à III.

\section{CONCLUSION}

L'exemple qui précède montre done que le problème de la régulation antomatique d'un système de transmission comportant l'accouplement d'ufi moteur thetmique et d'un moteur hydraalique n'est pas inabordable. Le problème peut comporter d'autres solutions en combinant erisemble des régulateurs à action directe et des r'égulatetrs à action indirecte.

\section{A. Renard,}

Ingénieur des Arts et Manufactures, Direcleur de la Papeterie de Poncé.

\section{ACADÉMIE DES SCIENCES}

\section{MÉCANIQUE ET ÉLECTRICITÉ}

Nouveau procédé de désélectrisătión des matières textí. les au moven des courants électriques de hate fréquencé. Nole de MM. J. PAILLET, F. Ducretet et $E$ Roger, présentée par M. d'Arsonval, séance du 6 mal's 1911.

On sait quie dans les différenles phases de la manútention dés matières textiles (laines, soies, colons, papiers, films), Il est né. cessaire, pour obtenir des produits de honne qualité et éviter les trop grands déchels de matière première, d'avoir recours à cortains moyeus qui présentent de graves inconvénients au point de vue de la salubrité ou de la sécurité des ouvriers.

Par suite de l'électrisation qui se produit pendant l'étiragre de la laine, par exemple, les fibres divergent et une notable partie se détache dé la mèche principale. Il en résulte un déchet très importànt, car la matière tombée est dépréciée.

En outre, lo til produil est de grosseur irrégulière et la casse devient fréquente ; la qualité du fil se trouve diminuée et le travail de l'ouvrier chargé de rattacher les fils devient excessif.

Le procédé d'hunidification généralement employé aujourd'hui ne remédic à cet inconvénient qu'aux dépens de la salubrité des salles, car toute aćration est interdite, les salles fortement surchauffées et les fenètres fermées par doubles crnisées.

En outre l'excès d'humidité sur les matières textiles augmente le collage des nèches sur les organes des métiors, ce qui est une nouvelle cause de déchets.

On a essayé dans le mème bul d'avoir recours à une charge électrique distribuée le long des métier's par des conducteurs isolés, cette charge étant produite par une bobine de Ruhmkorif ou une machine électrostatique, afin d'annuler l'électricité développée dans la matière textile; mais les fortes commotions ressenties all contact des coitducteurs dans le premier cas, pendant les différentes opérations de rattachage des fils et du nettoyagé, sont dangereuses pour les onvriers, sl l'on veut employer une certaine énergiè. Dans le sccond cas, la charge est très difficile à máintenir à une valeur rigoureuse pour assurer une heutralisation exacte sans produire un charge inverse.

Les courants de haute tension par une source de courant alternatif employés dans ce bui seralent extrèmement dangereux et inspplicables dang une usine:
Après différents essaís exécutés à Fourminies dans lès usines de iI. Paillet, nous arons obtenu le résultat cherché en utilisant les courants de grande fréquence à haute tension, tels qu'ils sont déj: appliqués en électrothérapie à la sunte des travaux de M. le Pra. fesseur d'Arsonval et plus récemment en télégraphie sans fil.

L'emploi de ces courants étant inoffensif, il est possible de mettre en jeu une grande énergie, sans qu'il en résulte ancun danger pour les ouvriers. Le procédé que nous émployons ac. tuellement et qui fonctionne régulièrement depuis le mois de mai rgto, utilise lá décharge oscillante de condensaleurs alimen. tés paŕ un courant alternatif survoltê au moyen d'un transforrinas teur. Lé dispositif ayant dónné le meilleur. résultat est celui quil est connu sous le nom de "résonnateur Oudin ", tcl qu'il \&i employé dans les stations de télégraphie sans fil. Les conducteur: aériens ou antennes sont ici distribués le long des métiers at. voisinage des malıères textiles à trater et sont garnis de place en place de pelits balais en fils métalliques très souples afin de ne pas hicsser les mains des ouvriers, el destinés à répartir la charye. aux polith précis oł elle dort être utilisée.

Le résonnateur Oudin se compose d'un grand solénorde à forle section ne comprenant qu'un petit nombre de spires composé dé deux sections dont on peut faire varier les longueurs au moyem d'in curseur mobile.

La décharge s'effectue à travers une section du circuit qui réagil sur les autres spires et sur les conducteurs de distribution par phénomiène de résonnance. L'étincelle de décharge jaillit entre les électrodes d'un éclateur ; le circuit inférieur du résonnateur est relié à la terre, et l'extrêmité supérieure communique arre les fils dériens. Uné bobine de self variable sert au réglage et l'accord du circuit primaire afin d'obtenir dans ce circuit le phé' nomène de résonance. Les radiations électriques sont ainsi utillséès dảis lés theilleures̃ conditions de rendement.

Lés résultats obtenus ont été officlellement constatés par le BH. reau de Conditionnement des soies et laines de Roubaix-Tourcoing et ont montré que la solidité à la traction, mesure de la limité de l'effort nécéssaire pour ameher la casse, était augmentée de 7 à 15 pour Ioo; dans certains cas, le chiffre a atteint 22 p. I00.

Quelles que soient les conditions d'humidité et de tempétature: les chiffres obtenus sont toujours en laveur de la méthodé nou velle. L'élasticité, qualité très importante en raison des opérationl postérieures du tissage, s'est trouvéc accrue dans la proportion dé 19 pour ıoo, et sur les laines de basse qualité, où le déchet es gónéralement très important, ce chiffre a atteint dans certain cas 28 pour rob.

Il implorte de considérer que ces résultats, qui procurent un bénélice sensible au filateur, ne sont pas obtenus aux dépens dit personnel ouvrier ; bien au contraire, lo nouveau systèniè permet de dimihuer; dafts une fotte proportion, le digré d'humidité el la tempếrature des salles de travail ; ces conditions mauvaisel étaient solivenit des sources de confhts entre les industriels et let inspecteurs du travail.

De l'avis même des ouvriers consultés à ce sujet, le nouveail procédé n'entrave on rien leur travail el soulage même leur efloti puisque les casses de fils deriennent moins fréquentes.

Il est aujourd'hui possible de voir dans l'usine de Fourmin les fenètres ouvertes et les salles largement aérées, chose inconnu? jusqu'ic de tous ceux qui connaissent les conditions ordinalirs du travail dans cette industrie.

\section{Recherches sur la constitution de l'étincelle électriquêt} Note de M. E. Caudrelier, présente par M. L. Boult? séance du 20 mar- 1911.

L'auteur a rendu compte précédemment $\left({ }^{*}\right)$ d'expériences do: tinées à étudier l'étincelle de décharge des transformateurs. If but de ses nouvelles recherches a été de déterminer l'influente de l'jonisation iniliale de l'air de la coupure.

La méthode employée était celle d'observation par soufflagêt

(*) E. Caudnelien, Comptes rendus, t. $x 48$, rgog, p. $1257 ; 1.15$ Ig10; p. 6ris. 